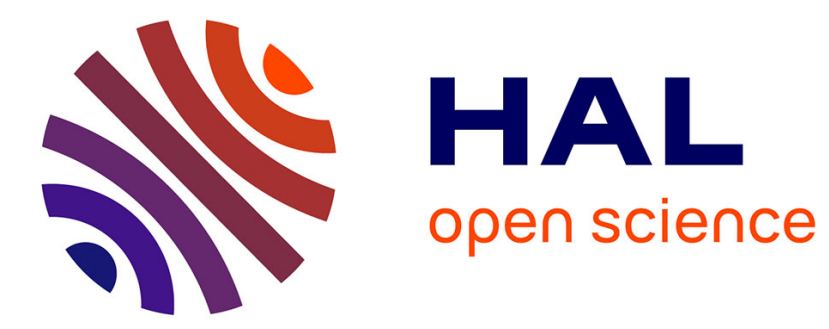

\title{
Les violences de la guerre d'Espagne
}

François Godicheau

\section{To cite this version:}

François Godicheau. Les violences de la guerre d'Espagne. Revue d'histoire de la Shoah, 2008, Violences de guerre, violences coloniales, violences extrêmes avant la Shoah, 2008/2 (189), pp.413-430. halshs-01552745

\section{HAL Id: halshs-01552745 https://shs.hal.science/halshs-01552745}

Submitted on 9 Oct 2017

HAL is a multi-disciplinary open access archive for the deposit and dissemination of scientific research documents, whether they are published or not. The documents may come from teaching and research institutions in France or abroad, or from public or private research centers.
L'archive ouverte pluridisciplinaire HAL, est destinée au dépôt et à la diffusion de documents scientifiques de niveau recherche, publiés ou non, émanant des établissements d'enseignement et de recherche français ou étrangers, des laboratoires publics ou privés. 


\title{
LES VIOLENCES DE LA GUERRE D’ESPAGNE François Godicheau
}

\author{
Mémorial de la Shoah | « Revue d’Histoire de la Shoah »
}

$2008 / 2 \mathrm{~N}^{\circ} 189$ | pages 413 à 430

ISSN 2111-885X

ISBN 9782952440974

Article disponible en ligne à l'adresse :

http://www.cairn.info/revue-revue-d-histoire-de-la-shoah-2008-2-page-413.htm

\section{Pour citer cet article :}

François Godicheau, «Les violences de la guerre d’Espagne », Revue d'Histoire de la Shoah 2008/2 (N¹89), p. 413-430.

Distribution électronique Cairn.info pour Mémorial de la Shoah.

(C) Mémorial de la Shoah. Tous droits réservés pour tous pays.

La reproduction ou représentation de cet article, notamment par photocopie, n'est autorisée que dans les limites des conditions générales d'utilisation du site ou, le cas échéant, des conditions générales de la licence souscrite par votre établissement. Toute autre reproduction ou représentation, en tout ou partie, sous quelque forme et de quelque manière que ce soit, est interdite sauf accord préalable et écrit de l'éditeur, en dehors des cas prévus par la législation en vigueur en France. Il est précisé que son stockage dans une base de données est également interdit. 


\section{LES VIOLENCES DE LA GUERRE D’ESPAGNE}

par François Godicheau ${ }^{1}$

La guerre d'Espagne présente de multiples formes de violences de guerre, que celles-ci aient eu lieu sur le front où s'opposaient deux armées - et où, en peu de mois, fut déployé un luxe de moyens qui en fait une guerre industrielle au même titre que la Grande Guerre -, ou qu'elles soient apparues à l'arrière, si tant est que, dans une guerre civile, la distinction entre l'arrière et le front soit vraiment pertinente. La particularité de l'Espagne, dans cette Europe de la première moitié du $\mathrm{Xx}^{\mathrm{e}}$ siècle, est qu'elle fut le théâtre d'une violence terrible, qui toucha profondément toute la société et mobilisa la majorité des habitants, une violence presque en tous points ressemblante à celle qui fut déployée dans les deux conflits continentaux, alors que le pays ne participa ni à l'un ni à l'autre. Elle échappa en particulier à l'influence directe du premier, au sens où l'on ne peut lui appliquer le raisonnement de Mosse résumé dans l'expression de "brutalisation " des sociétés européennes².

Même si les batailles furent loin d'y être aussi terribles que celles de la Grande Guerre et ne firent, en tout, qu'» à peine " 250000 morts, ce fut aussi une guerre de tranchées, dans laquelle est présente une mort de masse apportée par les pilonnages d'artillerie sur les fantassins lors de batailles d'usure, même si, en dehors d'une dizaine d'engagements importants, les fronts étaient calmes. Les exemples de

1. Maître de conférences à l'université de Toulouse-Le Mirail.

2. George L. Mosse, De la Grande Guerre au totalitarisme. La brutalisation des sociétés européennes, Paris, Hachette, 1999. Pour l'extension du concept au-delà du cas allemand, voir Stéphane Audoin-RouzeAu et Annette BECKER, 14-18. Retrouver la Guerre, Paris, Gallimard, 2000. Un point de vue historiographique dans Antoine Prost et Jay Winter, Penser la Grande Guerre. Un essai d'historiographie, Paris, Seuil, 2004, pp. 247-248. 
sauvagerie individuelle à l'encontre d'ennemis ne manquent pas, avec leur cortège de mutilations et d'acharnement sur les corps, et seul manque l'emploi des gaz - dont Franco disposait pourtant et dont il voulait faire usage. Les bombardements de villes ne constituant pas des objectifs militaires furent systématiquement employés par les franquistes, de Madrid à Barcelone en passant par Guernica.

Les victimes de ces bombardements, qui se comptent en milliers, ne sont pourtant qu'une infime partie des victimes de guerres occasionnées en dehors des fronts, aussi nombreuses que les victimes militaires. Du massacre de milliers d'hommes - mais aussi de femmes et d'enfants - par les troupes franquistes à Badajoz en août 1936, ou de celui de milliers de prisonniers " fascistes " de la prison de Madrid par les républicains, aux multiples assassinats d' " ennemis " dans les villages de l'un et de l'autre camp, les violences impliquèrent aussi bien des groupes que des individus, des troupes militaires que paramilitaires - les milices de volontaires dans l'un et l'autre camp. Il faut y ajouter la violence de genre, généralisée par les troupes franquistes (viols et tontes), ou celle exercée contre les enfants ; la violence des discours, où la promesse d'extermination et la déshumanisation de l'adversaire sont partagées ; et enfin les violences symboliques, dont les incendies d'églises et autres actes iconoclastes.

Par l'ampleur et la diversité des formes de violences qui s'y déployèrent, ce conflit de "l'entre-deux-guerres » est devenu une sorte de patron de la guerre civile, auquel sont rapportés d'autres épisodes, quand on cherche à les caractériser ${ }^{3}$. Par sa situation chronologique, elle s'intègre parfaitement à l'interprétation de la période 1914-1945 comme une guerre de trente ans. Cependant, les remises en cause actuelles de ses bornes chronologiques et les travaux sur ses violences mettent en question la notion de " violence de guerre ", notamment les rapports entre violences en temps de guerre et violences en temps de paix. Elles conduisent aussi à placer au centre de l'analyse les rapports entre identités collectives et institutions étatiques, et rendent enfin raison d'une violence qui, pour des raisons de politiques mémorielles, a longtemps posé des problèmes à l'historiographie.

3. Par exemple, au sujet de la France, par Olivier WiEWionKA, dans " Guerre civile à la française ? Le cas des années sombres (1940-1945) ", XX siècle, n 85, 2005/1, pp. 5-19. 
La guerre civile est considérée par les Espagnols comme un sommet de la violence politique dans l'histoire de leur pays, une histoire réputée violente, trop violente. Elle reste un problème face à auquel on tente de dresser un présent fait de démocratie et de " vivre-ensemble pacifique ${ }^{4}$ ". L'identité collective de l'Espagne démocratique d'aujourd'hui est très marquée par la vision de la guerre de 1936 comme une explosion de violence irrationnelle, " folie collective " dont les Espagnols auraient été saisis et par rapport à laquelle il importait, au moment de la Transition démocratique, de prendre le plus de distance possible, tant l'idée d'un retour de la guerre civile semblait possible. Ce sentiment de peur est très largement le résultat d'une politique de mémoire du franquisme tardif, consistant à opposer la stabilité du régime à la guerre, dont la réitération se serait profilée derrière chaque acte de l'opposition. Le caractère négocié de la Transition facilita la reprise par les premiers gouvernements démocratiques d'une vision voisine, celle d'une guerre essentiellement " fratricide ", où des horreurs auraient été commises de chaque côté et à l'opposé de laquelle il importait de construire la nouvelle Espagne ${ }^{5}$.

Cette idée, qui reposait sur une vision anthropologiquement fruste des Espagnols comme peuple " au sang chaud " et prompt à l'affrontement, fut bien sûr rejetée par les historiens de la démocratie, ce qui passa entre autres par l'organisation d'une discussion sérieuse sur le thème de la violence politique dans l'Espagne contemporaine, thème d'autant plus présent que la permanence du terrorisme semblait l'inscrire comme une manifestation de plus d'un " problème historique " national.

Un premier volume publié en 1994 sous la direction de Julio Aróstegui, sous le titre Violencia y politica en España, partait du caractère " significatif " de la relation entre ces deux termes au cours

4. L'expression originale est convivencia pacífica.

5. Sur ces questions, voir Paloma Aguilar, Memoria y olvido de la guerra civil, Madrid, Alianza Editorial, 1996 ; du même auteur, "Presencia y ausencia de la guerra civil y del franquismo en la democracia española. Reflexiones en torno a la articulación y ruptura del pacto de silencio ", in Julio Aróstegui et François Godicheau (dir.), Guerra civil. Mito y memoria, Madrid, Marcial Pons, 2006. 
des XIX et $\mathrm{XX}^{\mathrm{e}}$ siècles. Organisé de manière chronologique, il allait de la violence des traditionalistes carlistes à la fin du XIXe jusqu'à celle des nationalismes radicaux dans l'Espagne actuelle, en passant par l'action directe des anarchistes, la logique insurrectionnelle de certaines mobilisations politiques au début du $\mathrm{xx}^{\mathrm{e}}$ siècle et la violence d'État contre la subversion politique ${ }^{6}$. L'absence de la guerre de 1936 de ce livre est notable. Quelques années plus tard, un autre opus intitulé Violencia política en la España del siglo XX, sous la direction cette fois de Santos Julia, reprenait les mêmes thématiques, servies parfois par les mêmes auteurs, en ajoutant simplement la violence anticléricale et celle du patronat dans les conflits sociaux? La quasi-absence de la guerre de 1936 confirmait son statut de défi à la raison historienne.

En 1994, un premier chapitre tentait une synthèse des réflexions des sciences sociales sur la violence, passant très vite du binôme " violence et politique " à l'expression de "violence politique " et cherchant à en définir la "nature $"^{8}$. Six ans plus tard, l'objet ne semblait plus avoir besoin d'une définition ou d'une réflexion conceptuelle : selon l'introduction, il s'agissait d'étudier les idéologies, les langages et les pratiques de la violence politique qui a habité l'Espagne au Xx siècle. Autrement dit, la violence, c'est la révolution, la contre-révolution et le terrorisme. La violence politique est implicitement définie comme l'usage des armes dans l'arène politique et, de toute façon, comme un ensemble de pratiques et d'idées étrangères au présent démocratique. L'ensemble du livre semble reposer sur cette opposition entre un passé violent et un présent démocratique, mais marqué par une violence terroriste qu'il s'agit de rejeter dans le passé.

Qu'elle repose sur les disputes des sociologues et des politistes cherchant - dans un débat dont l'arrière-plan politique est souvent très visible - à délimiter ce qu'est la violence ou la violence politique, ou qu'elle parte du sens commun, la démarche consistant à étudier la " violence politique " comme une constante, dans ses manifestations

6. Revue Ayer, $\mathrm{n}^{\circ} 13$.

7. Madrid, Taurus, 2000.

8. Julio ArósteguI, "Violencia, sociedad y política : la definición de la violencia ", in Julio ARostegui (dir)., Violencia y politica, op. cit., pp. 17-55. 
diverses dans l'histoire contemporaine de l'Espagne ne semble, curieusement, pas opérante pour le grand déchaînement de violence de la guerre civile. Cela s'explique peut-être par la tendance de l'une et l'autre démarche à naturaliser la violence : les définitions sociologiques ou de sciences politiques peuvent-elles servir à l'historien quand " toute définition de l'action violente dépend des normes en vigueur qui donnent leur contenu à la notion ", lesquelles varient d'une époque à l'autre et en fonction des groupes, en particulier les normes du politique ${ }^{9}$ ? À ce propos, il est piquant de constater que dans un volume où l'ombre de la norme d'aujourd'hui prétend s'allonger sur tout le $\mathrm{xx}^{\mathrm{e}}$ siècle pour mieux servir la condamnation morale d'ETA, les articles illustrent en réalité à quel point perceptions, normes et légitimations de la violence ont variéi ${ }^{10}$.

Au-delà de la qualité des travaux contenus dans ces volumes, on sent, là encore, le poids des politiques de mémoire et l'économie morale de la Transition démocratique. Celles-ci ont d'abord pesé dans le sens d'un enclavement de la question des violences de la guerre civile, envisagée jusqu'à très récemment sous une rubrique au nom sans doute étranger : " la répression ". Au sein d'une histoire de la guerre civile constituant pratiquement un genre historiographique à elle seule, l'" histoire de la répression " a formé un véritable sousgenre $^{11}$. Sous le terme de "répression " étaient amalgamées les violences commises à l'arrière du front dans les deux camps, quelles que fussent les victimes - ainsi, dans le camp républicain, les violences à l'encontre des " ennemis fascistes " et la répression à l'encontre des révolutionnaires de l'été 1936 étaient mêlées. Par le jeu du parallélisme propre à la " réconciliation nationale ", le terme fut aussi appliqué à la terreur révolutionnaire de l'été 1936 dans le camp républicain et à l'ensemble de l'activité des tribunaux de ce même camp, qu'ils soient révolutionnaires ou gouvernementaux.

9. Olivier Fillieule, "L'émergence de la violence dans la manifestation de rue. Éléments pour une analyse étiologique ", Cultures et conflits, $n^{\circ} 9-10$, La violence politique dans les démocraties occidentales (ce texte est disponible à l'adresse : http://www.conflits.org/index212.html).

10. C'est en particulier le cas de l'article de Mercedes CABreRA et Fernando Del Rey Reguillo, "La patronal y la brutalización de la política ", dans Santos Julia, Violencia política, op. cit., pp. 235-288.

11. Pour une synthèse récente sur cette historiographie et les problèmes posés, voir José Luis LEDESMA, " El 1936 más opaco : las violencias en la zona republicana durante la guerra civil y sus narrativas ", Historia social, n 58, 2007, pp. 151-168. 
Cette veine historique particulièrement féconde a, grâce à de nombreuses études régionales, permis de sortir des estimations grossières et des légendes : une synthèse parue en 1999 permit même de projeter, à partir de l'étude de la majeure partie des provinces espagnoles, des chiffres globaux pour tout le territoire. Ainsi, la terreur franquiste aurait fait plus de 150000 victimes en comptant les années d'immédiat après-guerre, et les violences sur le territoire républicain quelque $50000^{12}$. Les auteurs distinguent une " terreur chaude " s'étendant sur les premiers mois de la guerre, jusqu'à la fin de l'année 1936, où se seraient produits la majorité des assassinats, et une " terreur froide " qui aurait pris la suite, faisant beaucoup moins de victimes au fur et à mesure qu'elle s'institutionnalisait. L'initiative serait passée, dans le cas franquiste, de l'armée et des milices à des tribunaux militaires et, pour le camp républicain, des milices et des comités révolutionnaires à divers avatars de tribunaux populaires de plus en plus contrôlés par le gouvernement, puis, à partir de la fin de 1937, à des tribunaux spéciaux beaucoup plus sévères que leurs prédécesseurs ${ }^{13}$.

Dans le camp franquiste, la terreur prit d'emblée un caractère systématique et fut dirigée contre tout le personnel politique républicain de gauche, le mouvement ouvrier et la franc-maçonnerie ; elle donna lieu à de véritables massacres, dont les victimes se comptaient par milliers, par exemple à Badajoz ou à Málaga, et elle fut assumée comme nécessaire et saine par toutes les autorités ; elle ne fut régularisée que par prudence vis-à-vis des possibles conséquences diplomatiques et dans le cadre d'une centralisation du pouvoir qui laissait moins de place aux spontanéités du terrain. Dans le camp républicain, la terreur révolutionnaire ne fut pas assumée par le gouvernement : elle fut attribuée aux multiples comitésgouvernements et aux milices populaires du début de la guerre, et bientôt à de fantomatiques " incontrôlés " dont l'invention reflétait le contrôle croissant du gouvernement sur la violence, privatisée

12. Santos Julia (coord.), Victimas de la guerra civil, Madrid, Temas de Hoy, 1999. Sur cet ouvrage, $c f$. François GodicheAu, "La represión y la guerra civil : memoria y tratamiento histórico ", Prohistoria, Rosario (Argentine), 5, 2001, pp. 103-122.

13. Pour une présentation de la justice républicaine en guerre, $c f$. François GodicheAu, $L a$ Guerre d'Espagne. République et révolution en Catalogne, Paris, Odile Jacob, 2004. 
dans les premiers mois, puis récupérée comme monopole étatique au fur et à mesure de la guerre. Cette mortalité est plus dispersée dans les villages, où les victimes de prédilection furent, dans l'ordre, les religieux, certains propriétaires - ruraux ou industriels - particulièrement conflictuels dans le passé récent et le personnel politique d'extrême droite.

Qu'elle insiste sur le parallélisme ou sur les différences entre les deux " répressions ", cette veine historiographique offre des caractéristiques toujours déterminées par les politiques de mémoire de la Transition : la plupart des livres réduisaient la " répression " aux assassinats et surtout se contentaient d'une description sommaire et d'un comptage des morts. L'établissement d'une comptabilité nationale rigoureuse et "scientifique " visait la fin des polémiques qui risquaient d'envenimer le présent. Le récit " définitif " de cette histoire tragique avait des objectifs pacificateurs que renforçait le parallélisme des descriptions, et l'asymétrie entre " répression d'État franquiste " et " répression spontanée républicaine " diluait les responsabilités entre des institutions disparues et des "incontrôlés " forcément, eux aussi, impersonnels.

Une fois réalisé ce travail comptable fastidieux et sans doute nécessaire, l'approche des violences de guerre a sensiblement évolué : d'une focalisation sur les seuls assassinats, les recherches se sont élargies d'abord à l'ensemble des violences pratiquées à l'encontre des combattants et des non-combattants et surtout à une activité véritablement analytique qui cherchait à dégager le sens de ces violences, à comprendre leurs ressorts. Aujourd'hui, ce champ n'est plus borné comme l'était l'» histoire de la répression " et participe pleinement du renouvellement, timide mais réel, des questionnements sur la guerre civile et, finalement, du désenclavement de l'étude de celle-ci au sein du contemporain espagnol.

Les violences anticléricales ont, par exemple, été abordées par l'anthropologue Manuel Delgado comme violences religieuses : écartant une histoire " rassurante " qui attribuait systématiquement les incendies d'églises et autres actes iconoclastes à une minorité par avance définie comme violente - les anarchistes -, il a cherché 
à comprendre comment des foules catholiques ont pu se retourner contre l'Église, ses représentants et ses symboles ${ }^{14}$. Il a reconstruit à propos de plusieurs villages les contre-rituels organisés par les iconoclastes en analysant cette violence comme liée à l'écroulement d'un monde et destinée à en faire naître un nouveau, à refonder la communauté sur les bases d'une action plus ou moins unanime. Le problème était que cette refondation manquait de mots capables de l'instituer et que, le lendemain, la violence se prolongeait, organisée, et servait, au même titre que les discours de guerre, à placer la limite entre amis et ennemis et à construire les identités respectives des uns et des autres ${ }^{15}$. Elle était donc difficile à assumer et fut enfermée dans la catégorie " violence révolutionnaire " ou "violence anarchiste ".

À l'inverse de la violence anticléricale et iconoclaste, celle qui fut très largement exercée par les vainqueurs contre les femmes républicaines fut dès le départ surchargée de sens par les discours vindicatifs à l'encontre de "la femme rouge ", symbole de "l'inversion des valeurs " qui caractérisait le camp ennemi. De la promesse de viols par les soldats maures aux théories pseudomédicales ou psychologisantes sur les femmes " dégénérées " du camp adverse, les violences de genre furent encadrées par des mots qui furent fondamentaux dans la construction de la figure de l'ennemi. Cette catégorie de violences de guerre servit aussi, pour les vainqueurs, dans le cas des tontes, à faire participer la communauté à la victoire, par la convocation de la foule au rituel d'humiliation qui entretenait la distinction entre le " nous " et le " eux $~^{16}$. Enfin, les violences dans les prisons franquistes contre des femmes considérées comme prises de guerre furent terribles et

14. Luces iconoclastas. Anticlericalismo, espacio y ritual en la España contemporánea, Barcelone, Ariel Antropología, 2001.

15. C'est ce que Rafael Cruz appelle " la construction sociale de la guerre " dans En el nombre del Pueblo. República, rebelión y guerra en la España de 1936 (Madrid, Siglo XXI, 2006, chap. 6, pp. 262 et suivantes), en reprenant la terminologie de Peter Berger et Thomas Luckmann dans La Construction sociale de la réalité (Paris, Armand Colin, 2006 [1966]).

16. Maud Joly, Histoire, mémoire et violence sexuée. En Espagne de la guerre civile au premier franquisme. Les tontes des femmes républicaines, mémoire de DEA, Institut d'études politiques de Strasbourg/université Rennes 2-CRHISCO, 2001 ; et, du même auteur, "Dire la guerre et les violences : femmes et récits pendant la guerre d'Espagne ", Mélanges de la Casa de Velázquez, 2007, $\mathrm{n}^{\circ} 37-2$. 
s'exercèrent aussi à l'encontre de leurs enfants en bas âge, lesquels mouraient en masse du fait des conditions d'incarcération atroces (sans compter les milliers d'enfants arrachés à leurs parents) ${ }^{17}$.

La réintroduction, par l'historiographie récente, de toutes les formes de violence dans l'étude du conflit a des conséquences sur la manière dont on peut envisager les violences de guerre. Tout d'abord, les distinctions entre violences régulières et irrégulières, légales et illégales, physiques et symboliques deviennent incertaines. En premier lieu, s'agissant d'une guerre civile, les normes et autres " lois de la guerre " sont " oubliées " et la tendance est à considérer l'ennemi comme l'on considère les combattants " irréguliers " et les partisans ${ }^{18}$. Dans la guerre civile, l'ennemi est partout : dans de nombreuses localités républicaines au début du conflit, le Comité antifasciste entretient à la fois les miliciens envoyés sur le front et des " milices de l'arrière ", celles précisément qui procèdent à l'épuration des "ennemis de l'intérieur ". De même, dans les premiers mois, les miliciens sont mêlés aux soldats de métier et la distinction entre civils et militaires ne fonctionne pas. En outre, dans ce conflit, le point de vue strictement militaire est une fiction : la terreur contre les populations civiles est utilisée comme une arme militaire et elle le restera par la suite dans la Seconde Guerre mondiale.

Enfin, les discours franquistes d'extermination de l'ennemi comme collectif, sa caractérisation comme une maladie à extirper du corps national, imprègnent aussi bien les actions militaires comme les bombardements ou les pilonnages d'artillerie sur l'infanterie adverse que les meurtres et les mutilations à petite échelle ${ }^{19}$. Souvent

17. Cf. Ricard VINYES, Irredentas. Las presas politicas y sus hijos en las carceles franquistas, Madrid, Temas de Hoy, 2002. Sur l'univers carcéral franquiste, voir la synthèse d'un grand colloque tenu à Barcelone en 2002 : Carme Molinero, Jaume SobrequÉs (dir.), Una Inmensa Prisión. Los campos de concentración y las prisiones durante la guerra civil y el franquismo, Barcelona, Crítica, 2003, dont le chapitre 8, écrit par Ricard Vinyes, a été publié en français sous le titre "L'univers carcéral sous le franquisme " dans Cultures et Conflits en 2004, $\mathrm{n}^{\circ} 55$ (ce texte peut être consulté à l'adresse suivante : http://www.conflits.org/index1568.html). Sur les enfants : Ricard VinYES, Montse ARMENGou et Ricard BeLIS (dir.), Los niños perdidos del franquismo, Barcelone, Plaza y Janés, 2002.

18. Cf. John Horne et Alan Kramer, 1914. Les atrocités allemandes, Paris, Tallandier, 2005.

19. Pour le discours médical sur l'ennemi, voir VinYES, ARMENGOU et BELIS, Los niños perdidos, op. cit, pp. 23-54. 
même, les violences se répondent : des meurtres de prisonniers par les républicains ont répondu à la nouvelle des massacres de Badajoz ou de bombardements par les franquistes ; à l'inverse, les sacas de prisonniers républicains ont eu lieu en " réparation " d'attentats contre des monuments religieux par les miliciens du camp adverse.

Il est donc très difficile de s'appuyer sur les normes de la guerre pour qualifier les violences observées, et le même problème se pose pour la distinction entre violences légales et illégales : le " refroidissement " de la terreur identifié par les historiens de la "répression " correspond à la fois à la diminution très importante de la mortalité et à l'adoption de procédures légales par les bourreaux. Mais ce processus est moins lié à l'imposition d'un ordre à un désordre qu'à l'institutionnalisation de la violence, qui s'accompagne d'une sélection plus rigoureuse des formes et des victimes, d'une plus grande attention portée au sens et à la légitimation des actes violents.

Pendant l'été 1936, l'État espagnol s'écroule et perd le monopole de la violence, mais aussi celui de sa légitimation : les corps d'armée et les milices des insurgés, de même que les milices populaires et les comités ouvriers qui leur répondent exercent des violences qu'ils tendent, peu à peu, à habiller de formes institutionnelles légitimatrices - de la proclamation sous forme d'avertissement aux ennemis de l'intérieur aux mises en scène de conseils de guerre ou de tribunaux révolutionnaires. Ensuite, dans les deux camps, on observe la (re)construction d'un État qui ne procède pas seulement par élimination de ces formes " chaudes " de la violence, mais par leur intégration : côté républicain par exemple, le souci des révolutionnaires de Lérida de légitimer leur action rencontre la volonté du gouvernement catalan de contrôler le territoire et de reconstituer un appareil judiciaire. Le même phénomène se produit pour l'institution policière, avec l'intégration plus ou moins rapide, à Madrid, Barcelone et ailleurs, des " milices de l'arrière " des premiers temps à une nouvelle police républicaine. Chez les franquistes, la création d'un appareil judiciaire - et même de l'ensemble de l'administration étatique - à partir de l'armée s'accompagne de l'intégration de la Phalange à l'appareil de répression et de surveillance. 
Le contrôle de la violence par l'État porte sur son intensité, mais aussi sur le sens qu'elle doit avoir. La réduction de la mortalité est liée au souci de donner à l'étranger l'image d'un territoire sur lequel règne l'ordre, sur lequel précisément l'État impose sa loi à tous, et même d'un territoire relativement homogène, uni derrière son gouvernement, où les " ennemis de la société et de l'État " sont sous contrôle ; bref, un territoire qui n'est pas déchiré par la guerre civile, mais mobilisé pour une guerre nationale ${ }^{20}$. En même temps, l'adoption de formes juridiques, même sommaires, et le monopole de la capacité de parler au nom de l'ordre public permettent de transformer la violence en " usage de la force " et de mieux contrôler la définition des cibles de cette violence. L'État en (re)construction cherche le monopole de la définition de l'ennemi et de la nature de la guerre. Exécution, tortures et brutalités sont d'autant plus facilement couvertes par le manteau de l'ordre public - notion dont l'ambiguïté apparaît clairement ici - que le contrôle démocratique des instruments de coercition étatique disparaît de l'ordre du jour.

Il faut, de ce point de vue, différencier clairement les deux camps : dans le camp républicain, même si l'apparition de tribunaux spéciaux militarisés prononçant de très nombreuses peines de mort - la plupart non validées en Conseil des ministres - et l'existence d'un service d'Information militaire, sorte de police politique liée à ces derniers, mettait la démocratie entre parenthèses, celle-ci restait un horizon d'attente de la République en guerre, et le maintien d'un relatif pluralisme politique fait de rapports de force au sein de l'" antifascisme " limitait les excès de pouvoir des agents contre les " ennemis de l'intérieur $n^{21}$. Dans l'autre camp, rien de tout cela : les sacas, les tontes, les tortures continuèrent longtemps, même après la fin officielle de la guerre le $1^{\text {er }}$ avril 1939, tout comme les expéditions punitives de phalangistes à la prison pour " casser du rouge " et les violences indi-

20. Cf. Nuñez SeIXAs, Xosé Manuel, Fuera el invasor ! Nacionalismos y movilización bélica durante la guerra civil española (1936-1939), Madrid, Marcial Pons, 2006 ; François GodICHEAU, "Les Espagnes de la guerre civile ", Atala, n 11, 2008 ; et " "Guerre civile", "Révolution", "Répétition générale" : les aspects de la guerre d'Espagne ", in Roger BouRDERON (dir.), La Guerre d'Espagne. L'histoire, les lendemains, la mémoire, Paris, Tallandier, 2007, pp. 89-105.

21. François GodicheAu, " La légende noire du SIM de la République dans la guerre civile espagnole et l'idée de contrôle politique ", in Le Mouvement social, 201, octobre 2002, pp. 29-52 ; et « Le poids de la guerre et l'évolution autoritaire de la République espagnole ", Humanismes. Revue du Grand Orient de France. La République espagnole, 70 ans après, Paris (à paraître). 
viduelles des agents étaient encadrées par des institutions qui organisaient elles-mêmes la violence. Le traitement des prisonniers dans les camps de travail, par exemple, et la mortalité particulièrement élevée de ceux que l'on a appelés récemment " les esclaves de Franco ", comme les déplacements de population et le massacre de villageois accusés d'aider les maquisards, relevaient d'une politique de guerre destinée à exterminer un ennemi déjà vaincu ${ }^{22}$.

Les milliers d'exécutions du printemps de 1939 perpétrées par les franquistes répondent donc à celles de l'été 1936. Mais ces assassinats nombreux posent un problème pour l'étude des violences de guerre : le conflit se termine officiellement le $1^{\text {er }}$ avril 1939, mais dès le lendemain, l'appel à éliminer l'ennemi intérieur est réitéré et les violences se prolongent longtemps après, pendant une décennie, en particulier dans la répression des guérillas. Ce sont précisément les études de la "répression " franquiste de 1936 aux années quarante qui ont rendu incertaine cette borne chronologique aval de la guerre $^{23}$. Mais la borne amont ne se porte pas mieux : dans une récente et nouvelle interprétation du conflit, l'historien Rafael Cruz a rappelé qu'au cours des premières semaines, pour les Espagnols de l'un et de l'autre camp, il n'y a pas de guerre ${ }^{24}$.

Pour les militaires et leurs alliés civils (miliciens phalangistes et traditionalistes), la prise d'armes correspond d'abord à une opération de maintien de l'ordre qui passe par le renversement du gouvernement et, pour certains, du régime ; cette opération prétend réagir à une

22. Fernando Mendiola Gonzalo et Edurne Beaumont Esandi, Esclavos del franquismo en el Pirineo. La carretera Igal-Vidángoz-Roncal (1939-1941), Txalaparta, 2006 ; voir aussi Gonzalo Acosta Bono ET ALII, El canal de los presos (1940-1962). Trabajos forzados : de la represión politica a la explotación económica, Barcelone, Crítica, 2004. Sur les guérillas, voir la synthèse de Mercedes YusTA, "Les Guérillas ou le prolongement de la guerre civile ", in Roger BouRDERON (dir.), op. cit., pp. 231-240.

23. La bibliographie étant très fournie, on se contentera, outre les titres déjà mentionnés de Ricard Vinyes et Carme Molinero, de citer Julián CASAnova, Francisco EsPInOSA, Conxita Mir, Morir, matar, sobrevivir. La violencia en la dictadura de Franco, Barcelone, Crítica, 2002 ; Michael RichARDS, Un tiempo de silencio. La guerra civil y la cultura de la represión en la España de Franco (1936-1945), Barcelone, Crítica, 1999 ; Mercedes YusTA, Guerrilla y resistencia campesina: la resistencia armada contra el franquismo en Aragón (1939-1952), Saragosse, PUZ, 2003 ; et Ángela Cenarro Lagunas, Cruzados y camisas azules. Los orígenes del franquismo en Aragón (1936-1945), Saragosse, PUZ, 1997.

24. Rafael CRUZ, En el nombre del Pueblo, op. cit., pp. 189 et sq. 
mobilisation révolutionnaire ou à une situation d'" anarchie ", en réalité fantasmée. En face, pour de très nombreux volontaires des milices ouvrières, la réponse au coup d'État est le premier acte de la révolution ; ainsi, par exemple, la masse des militants de la Confédération nationale du Travail (CNT) de Barcelone n'accepte de différer la prise complète du pouvoir qu'au lendemain de la prise de Saragosse, censée intervenir en quelques jours.

L'assassinat d'adversaires politiques considérés comme des ennemis ( " ennemis de l'ordre " ou " ennemis du peuple »), tel qu'il a lieu dans les premiers temps du conflit, ne relève pas en effet, en Espagne, des seules violences de guerre. De ce point de vue, la guerre civile est liée à un avant-guerre, non pas que les violences politiques d'avant-guerre préparent ou laissent présager la guerre civile, mais parce que la militarisation de l'ordre public a conduit à des situations de "guerre endémique " où la plus large violence d'État a été justifiée contre des fauteurs de trouble considérés comme des ennemis intérieurs.

Au lendemain de la Première Guerre mondiale, à partir de 1917 exactement, un cycle de mobilisations ouvrières et de répression eut lieu en Catalogne, pendant lequel les agents de "l'ordre " officiels - l'armée, la Garde civile et la police - et privés - " milice civique " du Somatén et " pistoleros " à la solde des industriels - affrontèrent directement la CNT et ses hommes qui, eux aussi, maniaient le pistolet et la bombe. Ce cycle prit l'allure d'une véritable " guerre sociale " entre le patronat catalan et les ouvriers, se solda par des centaines de morts et ne se termina que par l'instauration de la dictature du général Primo de Rivera. Mais ce serait une erreur d'y voir l'influence de la Première Guerre mondiale : même si la peur et l'espoir suscités par la révolution russe jouèrent un rôle, la crise d'un ordre public déjà traditionnellement sanglant et sa militarisation remontaient à bien avant la Grande Guerre ${ }^{25}$.

25. On en trouve un exposé détaillé dans deux livres de l'historien Eduardo GonZÁLEZ CAlleja, La razón de la fuerza. Orden público, subversión y violencia política en la España de la Restauración (1875-1917), Madrid, CSIC, 1998 ; et El Mauser y el sufragio. Orden público, subversión y violencia política en la crisis de la Restauración (1917-1931), Madrid, CSIC, 1999. Dans " La patronal y la brutalización de la política " (in Santos JuLIA, op. cit., pp. 235288), Mercedes Cabrera et Fernando del Rey Reguillo considèrent la violence du patronat à l'encontre des ouvriers comme une manifestation de la brutalisation dont parle Georges Mosse, 
Une bonne partie des violences de la guerre civile nous renvoient donc de nouveau à une histoire du temps long de l'Espagne contemporaine, une histoire au centre de laquelle se trouve, à mon sens, la question de l'ordre public : l'ambiguïté de cette notion, qui permet l'exercice d'un pouvoir sans limite à partir du moment où les perturbateurs de l'ordre sont officiellement désignés comme tels, a joué pendant la guerre de 1936, avant et après elle ${ }^{26}$. Depuis sa création en 1844, la Garde civile, principale force de l'ordre, a été une force d'occupation dans les campagnes, qui intervenait en ville, comme au cours de la troisième guerre carliste de 1872 ou dans les guerres coloniales, à Cuba, aux Philippines ou au Maroc ${ }^{27}$.

Nous ne sommes pas renvoyés pour autant à l'histoire plus ou moins naturalisée de la violence politique. Récemment, divers historiens ont formulé une proposition d'analyse de la question en terme d'action collective qui nous permet à la fois de sortir de la naturalisation que nous avons relevée et de pallier l'absence de la Première Guerre mondiale comme principe explicatif de la " brutalité " du conflit de $1936^{28}$. Celle-ci n'intervient ni directement, c'est une évidence, ni indirectement : en effet, l'explication par les idéologies ou par la militarisation de la vie politique ne tient pas, comme le montre l'histoire de la II République de 1931 à 1936. Avant le coup d'État de juillet 1936, les milices politiques sont très faibles en Espagne, pratiquement inexistantes ; et même au printemps 1936, durant les quelques mois de gouvernement de Front populaire, si la conflictualité politique se traduit par des dizaines de morts, ce sont les forces de l'ordre qui tuent le plus ${ }^{29}$.

(suite de la note 25) mais sans référence précise à un processus lié concrètement à la Grande Guerre ; cette brutalisation des mœurs ressemble fort à une " malédiction fatale " du XX⿳亠丷厂巾 siècle. Dans cet article, par ailleurs passionnant, le seul élément d'explication de l'usage du concept est donné page 239 par une référence vague à « la culture antilibérale et de l'extrémisme politique incarnés à partir de la Première Guerre mondiale par le bolchevisme, le conservatisme radical et le fascisme ".

26. José Carlos de BARTolomé Cenzano, El orden público como límite al ejercicio de los derechos y libertades, Madrid, Centro de Estudios políticos y constitucionales, 2002.

27. Diego López Garrido, La Guardia Civil y los orígenes del Estado centralista, Barcelone, Crítica, 2004.

28. Javier MuÑoz Soro, José Luis Ledesma et Javier Rodrigo (dir.), Culturas y politicas de la violencia. España siglo XX, Madrid, Siete Mares, 2005.

29. Cf. Rafael CruZ, En el nombre del pueblo, op. cit. pp. 166 et sq. Selon ses calculs, $43 \%$ des 262 victimes de cette période furent tuées par les forces de sécurité (parmi lesquelles la Garde civile est responsable de 58 \% des décès), 20 \% par des militants de gauche et $17 \%$ par des militants de droite, ce qui correspond aux proportions observées pour l'ensemble de la période républicaine. Sur la faiblesse de la militarisation politique, $c f$. pp. 143 et sq. 
De même, l'explication par les "idéologies radicales " ne fonctionne pas : le plus grand déploiement de violence pendant les cinq premières années de la République est la répression de la grève générale insurrectionnelle d'octobre 1934, et si les insurgés eux-mêmes tuèrent des Gardes, des prêtres et des militants d'extrême droite, ce ne fut pas le cas lors des soulèvements populaires de 1932 et 1933 qui furent pourtant, le plus souvent, réprimés dans le sang. Cette répression d'octobre 1934 vit l'État franchir un cap dans l'utilisation des forces armées : il envoya la légion espagnole qui appliqua les méthodes de la guerre coloniale, les plus violentes, celles qui supposent une déshumanisation de l'ennemi.

L'approche des violences comme composantes de l'action collective est tout d'abord intéressante en ce qu'elle intègre l'État et ses agents à l'ensemble des protagonistes possibles (dirigeants politiques, gouvernements, spécialistes étatiques ou privés de la violence) et le système de légitimation étatique dans la catégorie plus vaste des " définitions partagées " de la violence qui sont l'objet d'une lutte entre les protagonistes, certains invoquant d'autres légitimations que son caractère légal, comme la défense de droits menacés ou les " provocations " de la police. L'examen des espaces, des formes et des opportunités de l'action collective violente complète l'analyse qui vise à " savoir dans quelles conditions les confrontations non violentes basculent vers d'autres, de caractère violent, et vice et versa ${ }^{30}$ ".

Cette mise en perspective permet une classification des violences par ordre décroissant d'ampleur qui va de la guerre de conquête entre États, des génocides et des guerres civiles, des affrontements lors d'insurrections, des cycles de protestations, des processus de démocratisation et des changements de régime jusqu'à des mobilisations plus ponctuelles. Elle a l'avantage d'inclure les violences de la guerre civile et une partie de leurs formes et de leurs justifications dans un continuum en ne posant pas comme préalable ou comme élément de distinction radical le fait qu'il y ait ou non guerre.

30. Rafael Cruz, " Penser la violence collective en Europe dans une perspective historique ", in MuÑoz Soro, LedeSma et Rodrigo (dir.), Culturas politicas de la violencia, op.cit. 
Il reste que ce qui commence pendant l'été 1936 est bien une guerre et que s'y déroulent des phénomènes distincts et nouveaux : celui qui était la veille encore un adversaire politique devient ennemi, et l'exercice de la violence à son égard sert à délimiter les contours d'un " eux " et d'un " nous " qui, en définitive, sont incertains. Ce phénomène de construction en miroir des identités collectives date d'avant le 18 juillet 1936 et il a donné lieu à l'opposition croissante entre un " peuple républicain " et un " peuple catholique ", opposition pour laquelle la conquête du gouvernement servait à mettre en œuvre des politiques d'exclusions validées par l'État, un " ordre " qui n'était public que pour ceux qui faisaient partie de la bonne définition du peuple ${ }^{31}$.

Quand une partie de l'armée se soulève le 18 juillet 1936, ce cadre et cette dynamique se brisent avec l'écroulement de l'État et l'apparition d'un front au dessin imprévu, qui oblige les collectifs à se reconfigurer et les individus à choisir. Ce ne sont pas des identités toutes constituées qui s'affrontent : les premières semaines de conflit sont l'occasion de mobilisations collectives nouvelles et intenses où la construction des identités et leur institutionnalisation rapide sont des enjeux majeurs. C'est pour cette raison qu'il n'est pas possible de séparer ici les violences que l'on peut qualifier de symboliques des violences physiques contre les combattants et les non-combattants. Ces dernières sont chargées de sens pour ceux qui les imposent comme pour leurs victimes et les observateurs : il est donc essentiel d'étudier la manière dont ces actes sont vécus et assumés par les acteurs de l'époque - leur moralità pour reprendre la terminologie de Claudio Pavone - et celle dont sont construites les justifications et les légitimations à un moment où les pouvoirs et les institutions, en particulier étatiques, sont en pleine reconfiguration ${ }^{32}$.

L'explication de la violence et du passage à l'acte individuels peut trouver sa place dans une telle approche si l'on considère l'importance des identités collectives dans la construction des identités indi-

31. Cf. Rafael Cruz, En el nombre del Pueblo, op. cit.

32. D'autres ont souligné le caractère consubstantiel au massacre de l'affirmation radicale des identités, $c f$. Jacques SÉmELIN, "Analyser le massacre. Réflexions comparatives ", Questions de Recherche, $\mathrm{n}^{\circ} 7,2002$, CERI-FNSP, p. 10. 
viduelles et le caractère crucial des mots dans ce processus. Les mots ne font pas que décrire une réalité extérieure : porteurs de valeurs, ils sont des éléments essentiels de la construction sociale de la réalité ; ces valeurs sont instituées et les institutions tiennent ellesmêmes des discours qui réassurent de véritables " communauté de langage ${ }^{33}$ ". Deux historiens ont récemment caractérisé les premiers temps de la guerre civile comme des moments de "politique absolue " où des individus dont les cadres d'identité s'écroulent - dans une guerre qui commence comme guerre des mots - peuvent ressentir une urgence vitale à anéantir un autrui construit comme parfait négatif de soi ${ }^{34}$.

On peut tout à fait appliquer à ces premiers temps de la guerre civile ces mots de Jacques Sémelin : " La peur que suscite la menace de sa différence maléfique invite en fin de compte à le rejeter, ou même à le détruire : sécurité oblige. Et cette radicalité de la destruction du "eux" marque bien évidemment la toute puissance d'un "nous" triomphant de la mort. [...] L'identité donne le cadre dans lequel va prendre forme le processus de violence. La volonté de pureté durcit ce cadre identitaire en greffant sur lui une thématique du sacré, religieuse ou séculière, qui relève par là même de l'absolu. Le besoin de sécurité, en phase avec le contexte de crise qui a conduit au développement de cette formation imaginaire, rend urgent le passage à l'acte ${ }^{35}$."

Il y a ensuite divers degrés d'institutionnalisation de cette violence et de médiation entre les auteurs et leurs actes, la médiation étant comprise comme la capacité à conférer un sens à cette violence. Elle peut ainsi apparaître comme moins violente, acceptable dans le cas de l'application d'ordres officiels, de couverture par un discours officiel et, par exemple, dans le cas des femmes rasées, où la violence s'insère dans discours très agressif sur la " femme

33. $C f$. Berger et LuCKMANn, La Construction sociale de la réalité, op. cit., et Mary Douglas, Comment pensent les institutions, Paris, La Découverte, 1999.

34. Pablo SAnchez LeÓn et Jesús Izquierdo Martín, La guerra que nos han contado. 1936 y nosotros, Madrid, Alianza, 2006. Le concept est emprunté à Alessandro Pizzorno, " Politics Unbound ", in Charles S. MAIER (éd.), Changing Boundaries of the Political, Cambridge, Cambridge University Press, 1987, pp. 27-62.

35. Purifier et détruire. Usages politiques des massacres et génocides, Paris, Seuil, 2005, p. 72. 
rouge " reposant lui-même sur un machisme traditionnel. Ce processus peut aussi ne pas avoir lieu : l'explosion de violence anticléricale et iconoclaste de juillet-août 1936 n'est pas prise en charge efficacement par un discours correspondant à une identité collective en construction, mais seulement assumée par la propagande anarchiste qui ne touche qu'une partie des auteurs de ces actes.

Pour analyser les violences de la guerre d'Espagne, il faut donc tout d'abord sortir de la guerre, aller chercher du côté de la construction particulière de l'État contemporain dans ce pays, du poids de l'armée et de la militarisation de l'ordre public, et considérer que la violence de celui-ci est lié à des politiques d'exclusion qui introduisent une grande violence dans les mobilisations collectives. On peut ensuite observer, au cours de la guerre, à partir d'une explosion de violence terrible et multiforme, comment celle-ci participe de la reconfiguration et de l'institutionnalisation d'identités collectives en lutte et même, ce qui est proprement extraordinaire, à la construction de deux États qui tendent à transformer une " guerre civile " en deux "guerres de libération nationale ". 